\title{
Yanlışsız Öğretim Yöntemleri
}

\author{
Elif TEKIN \\ Anadolu Ontversitesi
}

Gulndimuzde, Amerıka Bırleşık Devletlen ve Kanada gıbı gelışmış ulkelerde òzel gereksınımls çocukların eğtımlerını kımı dunumlarda alt ózel sınuflarda, çoğt durumda ise normal sınıflarda gerçekleştırıldığı gorulmektedır Bu ortamlarda çalışan oğretmen ve eğitıcıler deneysel araştırmalarla geçerlılı̆̆ı sağlanan etkılı ve venmlı uygulamalı davranı analızı, sınıf kontrolu ve etkılı ögretım yontemlen gib değışı oğretım stratejılen arayışların surdurmektedırler

Bu çalışmada etkılı oğretım yontemle. nnden olan "yanlışsız oğretım yontertu" (епror* less teaching ya da near errorless teaching) turlennden tepkı ıpuçlarını (response promptıng) sunulduğu ơğretum yontemlen tanıtılmaya çalışılacaktıt Bu ơgretım yaklaşımı ıçın alanyazında "yakją̧ık yanlışsız ogretım" ya da "yanlışsız oğretım' yontemlen denılmektedır $\mathrm{Bu}$ ıncelemede yanlışsız oğretım tenmın kullanmak yeğlenmışır (om, Alberto ve Troutman, 1995 Wolery, Ault, Doyle, 1992)

Yanlışsız oğretım, becen ve kavramları en ıyı bıçınde ögrenmienı oğretım sırasında yapıłan hatalarla değıl, óğetım sırasındak olumlu yanıtar ve alıştımalar aracılığıyla ger. çekleşıı̆ı varsayımından hareket edılerek gelıştınimış bır yaklaşımdır (Wolery, Baıley ve Suga, 1988) Yanlışsız ơgretım yontemının uygulanabılmesı ıçın üç̧ temel örkoşulun bulunması gerekmektedır (a) Ogretmen, oğreteceğ becenyı ögrenctnın kapasıtesinı dikkate alarak belırlemelı ve sunmalıdır, (b) Ogretmen, gereklı durumlarda becen anajızı gelıştımmelı ve btr kerede sadece küç̧uk bur bólumun oğretul- mesın amaçlamalıdır, (c) Oğretmen, ögrencının ögrenmesını kolaylaştırıcı ıpucu, model olma, gıbı değışk oğretım stratejılenım uygulayabılır olmalıdir

Wolery ve diğ , (1988) yanlışsız öğretım yontemlerını ortaya çıkışın dört nedene bał̆lamaktadırlar (1) Yanlışsız oğretım yơntemlen etklı oğretım yontemlendır, (2) Yanlışsız ògretım yontemlerınde oğrencı hemen her denemeye doğru yant verdiğı ı̧̧ın oğretmen oğrencı arasında olumlu ılışkı gelışmesıne yardım eder, (3) Yanlışsız oğretım yŏntemı oturumlarında az sayıda olumsuz davranış sergılenır, (4) Ögrencı yapı̆ğ yanlışlardan çok az oğrendıł̆ ıçı yanlışsız oğgretım yöntemlen kullanılır

Bu incelemede yanlışsız oğretım yóntemlerının ozellıklenne, bu oğretım yontemlen uygulanurken dikkat edılmesı gereken noktalara ve bu yontemlenn etkılıklenne ılışknn açıklama ve araşurma bulgularına yer verilecektır Izleyen bótumde yanlışsız ogrretım uygulamalarinda yer alan temel terim ve kavramlar açıkanacaktır

\section{Kavramlar}

Yanlışsız ögretım yontemlenıın her bıлsı ıçın geçerlı olan hedef uyaran (target stumulus), kontrol edicı ve kontrol edıcı olmayan ipucu (controllıng and noncontrolltng prompt), denemeler arası sitre (tnal ınterval), tepkı aralı.্. (response interval) gıbı bazı kavramlar vardır Çalışmanın bu bolumunde bu kavramlar ve tanımlan omeklerle açıklanmaya çalışılacaktır 


\section{i) Hedef Uyaran}

Soru ya da ifade olarak sunulabilen hedef uyaran ogrenciye yanit vermesı gerektığtn anımsatmak uzere kullanılır Bır başka deyışle, hedef uyaran oğretmenun oğtencıye yoneltığı herhang bır yonerge ya da soru olarak tanımlanmaktadır Oğrencıye tepkıde bulunmasını sağlamak uzere kullanılacak olan hedef uyaran (a) becen yonergesı (task durection), (b) çevre duzenlemesı (envıronmental manpulatıon) ve (c) doğal olarak oluşan olaylardan (naturally occurng events) bur tanesı ya da bırleşımı kullanularak sunulur (Wolery ve dığ . 1992)

Becen yonergesunde ogrenciye davran\$̊ yerne getırmesı anımsatilırken nası yerıne getırmesı gerektığıne ılışkın bsr ıpucu verılmez Orneğın oğretım ya da yoklama aşamasında oğretmenın oğrencıye "Bana kırmız arabayı goster ", "Peçeteyı katja " bıçımındek kornutlan brer becen yonergesidir Omeklerden de anlaşlacağı gıbı oğtencıye sadece ne yapması gerekŭğ soylenır, oğrencının nasıl yapması gerektığıne ılışkın açılamada bulunulmaz

Çevre duzenlemesı, oğretmenun doğnidan sozel soru somnası yenne çevresel duzenleme yaparak oğrencının tepkıde bulunmasını sağlamasıdır. Orneğın, yemek masası hazırlama becerısı oğretılırken oğretmen gereklı araçgereçlen masasun uzerıne koyarak oğrencınn davranışı sergılemesıru sağlayabulır lien dere cede zthin ozuni olan oggrencilere becen ve kavram oğretımınde becen yonergesı sozel yonergeyle birlıkte sunulabılır Omeğın, ơgretmen yemek masası hazırlamak ıçın gereklı araç-gereçlen masanın uzenne koyarak "Haydı şımd, yemek masasını hazırla" bıçımınde becen yonergesin sunabtlır

Doğal olarak oluşan olaylarnn ogrretımde becen yonergesı olarak kullanulması durumunda ise becen ve kavramlar gunluk olaylar ıçindek oluşum sırası ıçınde oğretılmeye çalışılır Omeğın, oğrencıye dış fırçalana becenıı oğgeulırken yemek yeme eylemının bıtısıı oğrencyye dışı fııçalaması ıçın uyaran olur Kımı durumlarda ise hedef uyaran, ozel gereksınıml, oğrencının algılama, kendısıne sumulan yơnergelen takıp edebilme ve kendisınden serglemesı beklenen becerını kamaşıklığına gore hedef uyaran turlen bırleşturlerek de sunulabilır (Wolery ve diğ., 1992).

\section{2) Ipucu}

Yanlışsız ogrretım yontemlerının tumunde kullanilan br diger oge ıse ıpucudur Ik1 tur ıpucu soz konusudur kontrol edicı ıpucu ve kontrol edicı olmayan tpucu. Kontrol edici ıpucu oğrencının doğn tepłı vermesınu kesınleştıren ıpucu; kontrol edıcı olmayan ıpucu ise ogrencinun doğru tepkıde bulunma olasılıgın arturan ancak kesınleşıımeyen ıpucu olarak tanımlanı Mımık ıpucu, sozel ıpıcu, resımlı (ıkı boyutlu) ıpucu, model ıpucu, kısmı fizsksel ıpucu ve tam fizıksel ıpucu olmak uzere alu çeşı ıpucu vardır Örencıye uygun upucunun belırlenmesı ıçın ıse şu kurallar onenimektedır (a) Oğrencınin vucudu uzennde en az kontrol gerekturen ancak en etkılı olan ıpucu seçılmelıdır (least ıntrusıve method); (b) Gereklı durumianda ipucu turlen bırleştınlerek bırlıkte kullanılmalıdır, (c) Davranışla doğgudan Ilgiıiı ve en doğal ıpucu turu seçılmelıdır, (d), Ipucu sadece ogrencı dikkatın yoneitığı̆ dunımlarda sunulmalıdır, (e) Ipucu oğrencıyı destekleyıcı bıçımde ve oğretım atmosfer ıçınde sunumalıdır, (f) Ipucu olabıldığınce erken sılıkleşturimelıdır (Wolery ve dığ , 1988. Wolery ve dığ., 1992)

Yukanda siralanan kontrol edicı ipucu turlenne ılışkın omek vermek uzere oğrencıye ayakkaby bał̆lama becensını ogretılmekte olduğunu varsayalım. Bu amaçla oğgretmen oncelikle "Ayakkabin' gy" becen yonergessn sunar Bu becenye ilışkın olarak oğretmenın mımık ve jestlerle ogrenctye ayakkabıyı giymesın ıstedığı belırtmesiyle mimik ıpucu sunabilir Sozel kontrol edic ıpucu olarak ogrjetmen oğrencıye "Ayakkabıyı lkı ucundan tutarak arala ve ayak parmaklanndan başlayarak ayă̆ını ıçıne yerleşır." bıçımınde ıpucu sunabılır Wolery ve dığenlen, (1992) en az beş tur sozel ıpucu olduğunu ffade etmışlerdır (a) oğrencıye davranışı nasıl yenne getıreceğını soyleme, (b) oğrenciye davranuşın bar bolumunu nasil yerıne getreceğni soyleme, (c) oğencıye davranışı yerıne getırmesı ıçın bır kural sunma, 
(d) oğrencıye davråı̧ı yerıne getırmesı ı̧ın dolaylı yollarla açıklama sunma, (e) değışık alternatıflen anımsalma Omeğın, oğrenciye masadakı insan sayısı kadar bardag mutfak dolabından alarak masaya getırmest ogretlıyorsa "Bardakjarı geturır mısın"?" davranışı nasıl yerne getureceğın soylemeye, "Masadaka herkesın bur bardağı var mi" kural sunmaya, "Su ıçeceğımız zaman nereden ıçenz ${ }^{2 "}$ dolayh açıklamaya, "Bardak, tabak, çatal, kaşık, bıçak getırebilırsın" ise alternatıfler arasından anumsatma yaparak sozel ipucu sunmaya omek clarak venlebilır Resimlı ya da ik boyutu ipucunda ıse, yenne getımesı beklenen davraniş resmedılerek ya da yazılarak oğrencıye sunulur Orneğın, oğrenclye omlet pışırme davranışı oğretılırken oğretmen omlet pışırme davranışının becerı analızındekı her bır basamağın resmını çızerek oğrencıye nasıl omlet pışırıleceğın oğretebılır Ya da omlet pışırme davranışının becen analızındek her bır basamağın yazarak oğrencının bu basamağ takıp etmesı saglanabiltr Model olarak kontrol edicı spucunun sunulmasında ıse, oğretmen oğretılmek istenulen beceninn nasıl yenne getunidugon oğrencıye model olarak sergıleyebulır Omeğın, yemek sonrası masa sılme becensı ogretilırken oggretmen masayı kendısı sılerek yemek masasının nasıl sulındığmı model olarak gosterır.

\section{c) Tepkı Aralığı}

Tepkı aralığı. hedef uyaran ve ıpucu sunulduktan sonra oğencıntn yanıt vermesın beklemek uzere geçen sure olarak tanımlanmaktadır Omeğın, nesne ısmı tanıma becensı nın oğreuldığı bır çalışmada oğtetmenın "Bu nesnenın Ismı nedir ${ }^{*}$ becen yonergesın sunduktan sonra "Kapı" kontrol edicl Ipucunu sunarak 5 sanye sureyle oğrencının yarut vermesın beklemesıdır

\section{d) Denemeler Arası Sure}

Denemeler arası sure Ise, oğrencrye hedef uyaran ve ıpucu sunularak tepkı aralığı suresunce oğrencinn yamıt vermesı bekJendikten sonra yenı bur hedef uyaran sunuak uzere geçen suredır Orneğin, nesne ısmı tanıma becerısının ogrretıldı̆̆ı bır çalışmada oğretmenın "Bu nesnentn เsmu nedır ${ }^{n}$ becen yonergesın sunduktan sonra "kapı" kontrol edict ıpucunu sunarak 5 sanuye stureyle oğrencınun yanıt vermesın beklemest ve yanıt aralığından sonra oğrencının yanıtına bağlı olarak ıkıncı kez ayn ya da farklı hedef uyaranı sunmak uzere geçen suredir.

\section{Yanbışsı Öğretim Türleri}

Yanlışsı oğretım yontemlen genel olarak ıkı grupca toplanmaktadır (a) tepkı ıpuçlarının sunuldư̆u oğretım yaklaşımlan, (b) uyaran uyariamalarinun (stımulus modtficatıon) yapıldı ı̆ı oğretım yakjaşımları (Cıpanı ve Madigan, 1986; Cooper. Heron ve Heward, 1987)

Tepkı ıpuçlarının sunulduğu yaklaşık yanlışsız oğgretım yontemlerı (a) sabıt bekleme surelı oğretım yonterı (constant tıme-delay), (b) artan bekleme surelı oğreım yontemı (progressıve tıme-delay), (c) davranış oncesı ıpucu ve sınanta yontemı (antecedent prompt and test). (d) davmanş oncesı ipucu ve stitkleştimme yontemı (antecedent prompt and fade), (e) aşamalı yardım (graduated guıdance), (f) eşzaman)ı ıpucu ogretım yontemı (stmultaneous prompl/ng), (g) ıpucunu gıderek arturma yontemu (system of least prompt), (h) spucunu giderek azaltma yontemı (system of most prompt) olarak sekız grupta toplanabilı

\section{Yöntemi \\ a) Sabit Bekłeme Süreji Öğretim}

Yanlışsız oğtetım yontemlerınden olan sabıt bekleme surelı oğretım yontemınde hedef uyaranın ardından oğrencını doğru yanıt vermessn saglamak uzere kontrol edicu ıpucu sunulur Hedef uyaran ve kontrol edicı ıpucu arasında geçen sure sıstematık bıçımde artuniarak kontrol edıcı yardım sıltkleştırılır (Schuster, Stevens ve Doak, 1990). Wolery ve diğ, 1992)

Sabıt bekleme surelı oğretım yontemı uygulamasında gerçekjeştırlen ılk bırkaç oturumda hedef uyaran ve kontrol edıcı ıpucu 
eşzamanlı olarak sunulur ve bu oturumlara sıfır sanye bekleme surelı denemeler denır (Schuster, Gast, Wolery ve Gultunan. 1988, Schuster ve Gnffen, 1990. Schuster ve Griffen, 1991, Stevens ve Schuster, 1987, Wolery ve dığ, 1992) Omeğın, renk kavramının sabıt bekleme surelı ogrretım yontemıyle oğretıldığı bır çalışmada oğretmen kavramı oğretmek ıçın kullandığı nesneyı ışaret ederek "Bu ne renk" (orn, hedef uyaran, becen yonergesı vb) ve hemen ardindan "Kırmızi" (om, kontrol edıcı Ipucu) yanıtını sunar Belırl bır sayıda sıfır sanıye bekleme surelı deneme oturumu gerçekleştınldıkten sonra oğretmen becen yonergesı ve kontrol edıcı ıpucu arasında geçen sabıt sureyı beiıler ve tum uygulamalarda bu sure kadar bekler Bu surece sabıt bekleme surelı deneme oturumlan denr Becerı yonergesı ve kontrol edıcı ıpucu arasinda geçen sureye ıse ıpucunu gecıktırme aralı६ı denır (Schuster ve dig, 1988, Schuster ve Griffen, 1990, Schuster ve Gnffen, 1991, Stevens ve Schuster, 1987, Wolery ve diğ. 1992)

Sabut bekleme surelı ogretım yontemının ouzm (Auit, Wolery, Gast, Doyle ve Eszenstat, 1988), tlen derece zihunsel ozur (Browder, Morr's ve Snell, 1981, Mcllvane, Withstandley ve Stoddard, 1984), çok ozurluluk (KJeınent ve Gast, 1982), ogrrenme guçluğu (Sievens ve Schuster, 1987) ve gelışımsel genlik (Schœen ve Sıvıl, 1989) gıbı değısık ozur gruplayında yer alan çocuklara ogretım yapmakta etkılı olduğ kadar okul oncesı donemden yetışkınlık donemıne kadar değışık yaş gurubundakı kışılere becen ve kavram oğretmekle de etkalı olduğ gorulmektedır (Kleınert ve Gast, 1982, Schoen ve Sivil, 1989)

Sabıt bekleme surelı oğretım yontemınn oğretılen becerı ve davranışlar bakımından hem tek-basamał. J (McIlvane ve dig. 1984) hem de zıncirteme davranışların (McDonnel ve Ferguson, 1989, Schuster ve dið̆ , 1988) oğretımınde etkılı bır yontem olduğuna ılışkın bır çok arastırmaya rastlanmaktadır

Wolery ve dig, (1992) sabit beklomict sureh ởretım yonetımınun etkılı bıçımde kullanılabılmesını saglayabılmek uzere sekız basa- maklı bır model gelıştırmışlerdır (a) ogrrencının tepkıde bulunması ıçın ıpucu olacak uyaranı belırteme, (b) kontrol edicı ıpucunu belırleme, (c) ogrencının yardımı bekleme becensını belırleme, (d) 0-sanuye bekleme surelı deneme oturum sayısın belırieme, (e) ıpucunu geciktır me suresını belırleme, (f) oğrencı davranışlarına ne şekılde yanıt venleceğını belırleme, (g) ver kayıt yontemını belırleme. (h) uygulama, kayıt etme ve oğrencının gosterdığı perfonmansa gore uygulanan ogretım yonteminde gereklı gorulen değışıklıklen yapma

Sabıt bekleme sưrelı ogretım yontemıle çarpma ışlemı (Alı-Cybriwsky ve Schuster, 1990, Mattungly ve Bott, 1990), sozcuk okuma becenst (Gast, Doyle, Wolery, Ault ve Baklarz, 1991), sozcuklentn antamların oğrenme becerıs] (Schuster ve diğ, 1990) gibı tek basamakJı davranşlaria bırlıkte, ılen derecede żhın ozurlu ergenlerle yemek hazırlama becensı (Schuster ve dıళ , 1988), oğretulebtlır zuhın ozurlu çocuklara yemek tanfi takıp ederek soğuk ıçecek hazırlama becensı (Schuster ve Gnffen, 199l) gibı zuncırieme becen ve davranışlann da ogretıldığı gorulmektedır.

Dıger taraftan, bu ogretım yonetımının verımlılı̈̆ıne ılışkn yurutulen çalışmalarda oğretım sırasında gerçekleşen hata yuzde duze yının çok duşuk olduğu (\% 4-5), araç-gereç hazırlama ve uygutama açısındas kolay bır yontem olduğu, oğretmen ya da uygulamaçınn fazla hazıriı yapmasını gerektırmedıg gibı venmlılık etmenun ọlçen değşken]er açısından oldukça ekonornk bır oğretım yontemı olduğu da gorulmektedır

\section{b) Artan Bekleme Süreli Ögretim Yöntemi}

Artan bekleme surelı ogretum yonteminde hedef uyaran ve kontrol edic yardım arasındakı sure sıstematik olarak artırılarak oğrencınun doğru tepkıde bulunması sağlanır Hedef uyaran ve kontrol edicl yardım arasında geçen sure sıstematık bıçımde artırnlarak kontrol edict yardım sılıkleştırlır (Wolery ve dığ. 1986, Wolery ve dig. 1988) 
Artan bekleme surelı ogretım yonteminde ogrretmen llk bırkaç denemede hedef uyaran ve kontrol edıcı ıpucu eşzamanlı olarak sunar Ogrtencının bu denemelerde verdığ yanıta baglı olarak ogretmen hedef uyaran ve kontrol edıcı ıpucu arasinda geçen sureyı I sn, 2 sn gıbı kısa aralıklarla artırır Oğrencı ıstentlen yanıt sununcaya değın denemelere devam edılır (Wolery ve diğ, 1986)

Artan bekleme surelı ogrretım yontemının ouzm (Gardıll ve Browder, 1995, Venn, Wolery, Werts ve Morns, 1993, Wolery, Gast, Kurk ve Schuster, 1988), hafif zıhunsel ozur (Walls, Haugth ve Dowler, 1982) orta zuhinsel ozur (Colluns ve Stunson, 19941995, Doyle, Schuster ve Meyers, 1996, Fredenck Dugan, Test ve Warn, 1991), çok ozurluluk (Goetz, Gee ve Sator, 1983), ogrenme guçluğu (Ault, Wolery, Gast, Doyle ve Martın, 1990) ve gelışımsel genlık (Gardıll ve Browder, 1995, Lucıano, 1986) gıbı değışık ozur gruplarında yer alan çocuklara oğretım yapmakt3 etkılı bır yontem olduğu gorulmektedır

Artan bekleme surelı ogretım yontemı nın de ğı̧ık yaş gruplarına becerı ve davranış ogretımunde etkılı bır yontem olduğu da araş urma bulgulanyla desteklenmıştır Ogretım yontemıni okul oncesı donemde (Charlop, Schresbman ve Thıbodeau, 1985, Venn ve diğ, 1993), ılkokul donemınde (Ault ve dığ, 1990, Barrera ve Sulzer-Azaroff, 1983, Doyle ve di 1996, Wolery ve diğ, 1988,) ortaokul ve lise donemınde (Farmer, Gast, Wolery, ve Win terlıng, 1991, Colluns ve Sunson, 1994-1995) ve yetışkınlerde (Fredenck-Dugan ve dığ, 1991, Gradıll ve Browder, 1995, Zane, Handen, Mason ve Geffin, 1984) etkılı bıçımde kullanıdığ gorulmuştur

$\mathrm{Bu}$ ogretım yontemının sozcuk ve bu sozcuklerın kısaltmaların oğretme (Ault ve dığ, 1990), ylyecek maddelerının fotoğraflarindan ısımlenın ogretme (Doyle ve diğ, 1996), resımlere bakarak nesnelenn ısımlerın oğretme (Wolery ve dığ, 1988) gibı tek-basamaklı becen ve davranışlarla, hesap makınası kullanarak alışverış yapma (Fredenck-Dugan ve dığ , 1991), bağımsız olarak kuçuk boyutlu alışverış yapma (Gardıll ve Browder, 1995) ve yatak yapma (Snell, 1982) gibs zincırleme becenterin oğretımınde etkılı olarak kullanıldığı gonulmek tedir

Wolery ve dugerlen (1992), artan bekłeme surelı oğretım yontemının etkulı bıçımde uygulanabıtımesı ıçın aşağıda sıralanan dokuz basamaklı ogretım planını onermışlerdır (a) ogrencının tepkıde bulunması ıcın ıpucu olacak uyaram belıreme, (b) kontrol edıcı lpucu belırleme, (c) ogrencının ıpucunu bekleme becerisıne sahup ofup olmadığn belırleme, (d) 0-sanye bekleme surelı deneme sayısını belı' leme, (e) ıpucunu gecıktırme aralık suresım beiırleme, (f) upucunu gecıktırme arahk suresinı artırma planını belırleme, (g) oğrencı tepkılen ne venlecek uygun tepkilern belırleme, (h) hedef davranışa bağlı olarak uygun ver toplama sistemını bejurleme, (1) uygulama, kaydetme ve oğrencının gosterdığı performansa ılışkın değı şıkıklerde bulunma

\section{c) Davranı̧ Öncesi Ipucu ve Sınama Ögrretim Yöntemi}

Davranış oncesı ıpucu ve sınama yontemınde, başlangıçta oğretmen kontrol edıcı ıpucu ve hedef uyaranı bırlıkte sunar $\mathrm{Bu}$ davranış oncesı ıpucu belı sayıda deneme ya da oturumda sunulur Deneme ya da oturumlardan sonra oğretmen davranış oncesı tpucunu tamamen ortadan kaldırarak ogrencının sadece hedef uyaran sunuilduğunda doğru tepkıde bulunup bulunmadığınt sınar (Wolery ve dığ , 1992 , Wolery ve dı

$\mathrm{Bu}$ yontemde uygulamacı ya da oğretmenın dıkkat etmesı gereken nokta beltı sayıda kontrol edict ipucu sunulduktan sonra kontrol edıcı ıpucunun tamamen ortadan kaldırılması dır Davraiış oncesı ıpucu ve stnama oğretım yontemının otızm (McGee, Kraniz ve $\mathrm{Mc}$ Clannahan, 1984, Salnato, Strann, Lefebvre ve Rapp, 1987), hafif zihınsel ozur (Sindelar, Bursuck ve Halle, 1986), Ilen zihınsel ozurfuluk (Peterson, Austın ve Lang, 1979), fizıksel ozurluluk (Hardtman, Goetz, Reuter ve LeBlanc, 1975) ve ogrrenme guçluğu (Cooke ve Appollonı, 1976) gıbı değışık ozur gruplarında 
yer alan çocuklara oğretım yapmakta etkılı bır oğretım yontem olduğu gorulmektedır

Oğretım yontemının değışı yaş grup larına becen ve davranış oğretımınde etkılı bır yontem olduğu da araştırma bulgularılya desteklenmuştur Okul oncesı donemde (Fınk ve Bnce Gray, 1979, Katz, Goldherg ve Shurka, 1977. Shaptro ve Sheridan, 1985), Ilkokul doneminde (Koegei, Dunlap, Ruchman ve Dyer, 1981) ve yetışkunlerde (Wacker ve Greenbaum, 1984. Weich, Nıetupsk ve Hamtre - Nıetupskı, 1985) etkılı bıçımde kullanıldığ gorulmuş̧ur

Ayrıca bu oğretım yontemıyle totoğaflara bakarak nesne istmlerun soyleme (Rowan ve Pear, 1985), sozcuk okuma (Fink ve Brice Gray, 1979) gıbı tek basamaklı becerlenn ve telefon kullanma (Smuth ve Meyers, 1979), bısıklet kullanma (Welch ve diğ, 1985), ba ğımsız hareket etme (Sànato, Stran, Lefebvre ve Rapp, 1987) gibı żncırleme becen ve dav ranışların da etkılı bır bıçımde oğretıldığı gorulmektedır

Wolery ve diğerlerı (1992), davrauş̧ oncesı ıpucu ve sınama oğretım yontemını etkılı bıçımde uygulanabılmesı ıçın aşağıda sıralanan yedı basamaklı oğretım planın onermışıerdır (a) ogrencının tepkıde bulunmasıı ıçın ıpucu olacak uyaran beiırleme, (b) kontrol edıcı ıpucunu belıtteme, (c) davranış oncesı ıpucunun nasıl sunulacağını belırleme, (d) sınama yontemın belırleme, (e) tepkı aralığı suresını belırle me, (f) ogrencı tepkulerine sunulacak uygun tepkulen belırleme, $(\mathrm{g})$ uygulama, kaydetme ve oğrencınn gosterdı ğı performansa liışıın değısikikiterde bointoms

\section{d) Davranı̧ Öncesi Ípucu ve Silik-} leştirme Örretim Yöntemi

Davranış oncesı ıpucu ve sılıkleştırme yontemınde, ogretrnen ya da uygulamacı kontrol edıcı upucu ve hedef uyaran birlıkte sunar ve zamanla davranış oncesı ıpucunı ortadan kaidırr $\mathrm{Bu}$ yontemde kontrol edıc spucunu ortadan kaldırma surecı sıstematık bıçtmde belırtenmemıştır, ogretmenın ya da uygulama cının oznel goruşune dayalı olarak gıderek ortadan kaldırnlır (Wolery ve diğ , 1992, Wolery ve dığ, 1986) Bır başka deyışle, omeğın ıpucunu gıderek azaltma oğretım yontemunde olduğu gibı kontrol edıcı ıpucunu azaltmak uzere bir olçut belırlenmez Davranış oncesı ıpucu ve sılıkleşturme oğreum yontemının hafif zihunsel ozur (Frank ve Wacker, 1986), orta zhhunsel ozur (Crouch, Rusch, ve Karlan, 1984), tlen zhunsel ozurluiluk ve çok ozurluiluk (Gee, Graham, Sallor ve Goetz, 1995, Stenberg, McNemey ve Pegnatore, 1985), otzm (Barrera, Lobato-Barrera ve Sulzer-Azaroff, 1980) ve gelışıtnsel genlik (Deckner ve Blantor, 1980. Salısbury, Wambold ve Walter, 1978) gibı değısık ozur gruplartnda yer alan çocuklara ogretım yapmakta etkılı bır oğretım yontemı olduğu gorulmuştur

Bu ợreum yontemule hemen tum yaş gnuplarındakı kışılere okuloncesı donemden yetışkınlı̆ge kadar- becen ve davranış oğretımınde etkılı ogretım yapıImaktadır Okul oncesı donemde (Barrera ve đi. , 1980), ılkokul doneminde (Koegel ve Rıncover, 1977) ortaokul ve lise doneminde (Deckner ve Blanton, 1980) ve yeuşikınlerde (Crouch ve dığ, 1984) etkılı bıçımde kullanuldı ğ gorulmuştur

Davranış oncesı ıpucu ve stlıkleştırme yontemını kullanildiğı bеcenlenn buyuk çoğunluğu yonerge takıp etme (Whutrnen, Zakaras ve Chardos. 1971), vucudun bolumlenIn tanıma (Koegel ve Runcover, 1977) gibı tekbasamaklı davranışiar olmasına karşın bağımsız yemek yeme (Knapcyzk, 1983), karşıdan karşıya geçme (Marchuettu, McCartney, Dran, Hooper ve Dix, 1983) gibj kmu zuncirleme davranışıann oğretımınde de etkılı olarak kullanıldığ gonumektedır (Gee ve dt

Wolery ve digerlen (1992), davranı oncesı tpucu ve sılıkleştırme oğretım yontemının etkılı bıçınnde uygulanabılmesı ıçın aşağıda stralanan yedı basamaklı ogretım planın onermışlerdır (a) oğrencının tepkıde bulunması ıçın tpucu olacak uyaranı belıleme, (b) kontrol edic ıpucunu belirleme, (c) ıpucunu silıkleşurme yontemını belırleme, (d) tepk aralığın belırieme, (e) ogrencı tepkllerine sunulacak uygun tepkıler belırleme, (f) hedef davranuşa bağhı 
olarak uygun ven toplama sıstemın belırleme, (g) uygulama, kaydetme ve oğrencının goster dığı performansa ılışkın dex̧ışıkltklerde bulun ma

\section{tem}

\section{e) Aşamalı Yardım Ögrretim Yön-}

Yanlışstz oğretım yontemlertnden bır dığen olan aşamaiı yardım ợretım yontemınde oğretmen oğretıme kontrol edıcı ıpucu sunarak başlar ve zamanla kontrol edıcı ıpucunu ortadan kaldırır Bu oğretım yontemı davranış oncesı ıpucu ve sılıkjeştıme oğretım yontemıne çok fazla benzemekle birlıkte, bazı açılardan dığer yontemlere de benzemektedır Bu yontemı tum diger ogretum yontemlerinden ayıran nokta, oğretmenın oğrencının gosterdığı performansa bağlı olarak kontrol edıcı spucu turu ve miktarna ıışkın anlık kararlar almasıdır (Wolery ve dığ , 1992) Oğretmen ya da uygutamacı oğre tım sırasında eğer kontrol edıcı ıpucunun oğrencısın doğsu tepkıde bulunması ı̧̧ı gerekls olduğuna inanussa kontrol edicı ıpucunu sunmaya devam eder, oğrencının doğru tepkıde bulunması ıçın kontrol edıcı ıpucunun gereklı olmadığna ınanıssa kontrol edıcı ıpucunu ortadan kaldırır

Aşamalı yardım oğretım yontemıntn otuzm (MacDuff, Krantz ve McClasnahan, 1993), hafif zuhınsel ozur (Schloss, Alexander, Hamıng ve Wnght, 1993), orta zihınsel ozur (Stumbert, Minor ve McCoy, 1977) ve then derece zihunsel ozur (Cupan, Augustune ve Blomgren, 1982, Paisey, Whitney ve Moore, 1989. Schoen, 1986, Singh ve Milıchamp. 1987), gok ozurluluk (McKelwey, Sisson, Van Hasselt ve Hersen, 1992, Reese ve Snell, 1991, Sisson, Kliwein ve Van-Hasselt, 1988), ve gelışımsel genlık (Mc Kelwey ve dı̌̆, 1992, Stsson ve duğ , 1988, Young, West, Howard ve Whitney, 1986) glbı değışı ozur gruplarında yer alan çocuklara oğretım yapmakta etkılı brr ởretım yontemı olduğu araştırma bulgulanyla desteklenmıştır

Bu ogretım yontemının okul oncest donemde (Sumbert ve dif , 1977, Young ve duğ, 1986), llkokul doneminde (MacDuff ve dır , 1993, McKelwey ve dığ, 1992, Reese ve Snell, 199I. Sisson ve dığ, 1988, Stumbert ve dığ 1977) ortaokul donemunde (MacDuff ve dı̊. 1993) ve yetışkınlerde (Cıpanı ve dığ, 1982, Parsey ve diğ. 1989, Singh ve Mılıchamp, 1987) etkılı bıçımde kuilanıldığı gorulmuştur

Aşamalı yardım oğretım yontemıyle kendıne zarar verme davranı̧̧ının azalılıması (Paisey ve diğ, 1989), bağımsız oyun oynama davranışının kazandırılması (Sıngh ve Mıllıcamp, 1987) gubı tek basamaklı sosyal davranışlarda de ğı̧ıłlık yapılabıldı ğ gıbı, yemek hazırlama (Schloss ye dis, 1977) ve bağımsız gıyınme (McKelvey ve diğ, 1992, Reese ve Snell, 1991, Sisson ve dir. 1988, Young ve dığ , 1986) gibı zıncırleme becenlerin ơgreumının de elkılı bır bıçımde gerçekleştı̆ı gorulmektedir

Wolery ve dığerlerı (1992), aşamalı yardım oğsetım yontemının etkılı byçınde uygulanabılmesı ıçın aşă̌ııda sıralanan alt basamaklı bır oğretım pianı onermışierdır (a) oğrencımın tepkıde bulunması ıçı ıpucu olacak uyaran belırleme, (b) kontrol edict ipucunu beltrteme, (c) spkcunu silikleştıme yontemın belırleme, (d) oğrenct tepkı!erıne sunulacak uygun tepkıler betırleme, (d) hedef davranışa bağlı olarak uygun verı coplama sıstemın belırleme, (f) uygularna, kaydetme ve oğrencının gosterdığı performansa llışkın değışıkliklerde bulunma

Davranış oncesı ıpucu ve sınama, davranş̧ onceș upucu ve silıkleştimne ve aşamaiı yardım ợreum yontemienne ıışkın gerçekleşurılen alanyazın taramasında ozellıkle geçmış yıltarda, kımı araştırnacı ve uygula macıların bu yontem kullandığı, kullandıkları yontemlen bu isımlerle ısımlendımedıklen ancak, uygulama surecım ayrmtıli bıçımde açıkladıkları gorulmuştur.

\section{f) Eşzamanh İpucuyla Öğretrm Yön-} temi

Yanlışsız oğretım yontemlerınden eş zamanlı ıpucuyla oğretım yontemı davranıs 
oncess ıpucu ve sinama oğretım yontemmnn (antecedent prompt and test procedure) ststematık bur bıçımı olup (Wolery ve dıg, 1992), etkık oğretım alanyazınında son yıllarda yer alan ancak hajen yeterınce araştırılmamuş bır sıstematık ogretım yontemıdır (Gıbson ve Schuster, 1992, MacFariand-Smıth, Schuster ve Stevens, 1993, Schuster, ve Gnffen, 1993. Schuster, Griffen ve Wolery, 1992)

Eşzamanlı ıpucuyla ogretım yontemınde, kontrol edicı upucu hedef uyaran hemen ardindan sunulur ve ogrencı kontrol edicl ıpucunu model alır Bu yontemde her denemede kontrol edics spucunun sunulmasi nedentyle oğrencıye bağımsız ofarak tepk veme olanağı hıçbır bıçımde sunulmadığı ıçın uyaran kontrol transferının-kontrol edicı ıpucundan ayırdedicı uyarana gerçekleşıp gerçekleşmedığı yoklama oturumlarında anlaşılır (Gıbson ve Schuster, 1992, MacFarland-Smith ve diğ, 1993, Schuster ve Griffen, 1993, Schuster ve dığ. 1992)

Eşzamanlı ıpucuyla oğretım yontemı davranış oncesı ıpucu ve test yontemıne çok fazla benzernekle bırlıkie ıkı açıdan onemlı farkjılıklar gostenr Bırıncı onemlı larklılık, eşzamanlı ıpucuyla oğretım yontemınde kontrol edıcı ıpucunun sureklı kullanılması, davranış oncesı ıpucu ve sınama ogretım yontemınde ise kontrol edici ıpucumun zaman zaman kullanılmasıdır Bu nedenle de. oğretım sırasındakı hata duzeyı dığer yaklaşık yanlışsız oğretım yaklaşımlarına gore daha yuksektır (\%5) Ikınct onemlı farkłılık ıse, eşzamanlı ıpucuyla oğretım yontemınde oğrencının performansını belırlemek uzere duzenlenen yoklama oturumlarının sadece ogrretım oturumu başlamadan yapılmasıdır Davranıs oncesı ıpucu ve sınama ogretım yonteminde yoklama evrest istenılen hemen her zaman, genelikke oğretımden hemen sonra gerçekleşiırılebılır (Gıbson ve Schuster, 1992, MacFarland-Smith ve dig, 1993, Schuster ve Gnffen, 1993, Schuster ve dug, 1992)

Eşzamanlı ıpucuyla oğretım yonternı sabıt bekleme surelı ogretım yontemtyle ortak bazı ozellıkler taşır Sabıt bekleme surelı oğreum yontemının ılk burkaç oturumu sifır sanye sabıt bekleme surelı oturumuar bıçımınde uygulanur (kontrol edicı ipucu becen yonergesinn hemen ardundan sunulur) ve bu bırkaç oturum dan sonra becen yonergesi lle kontrol edicı ıpucu arasında belırlı bir sure birakılarak ogrencıye bağımsız olarak yanıt verme şansı tanınır Eşzamanlı ıpucuyla oğretım yontemıyle oğretım yapilırken bu sure bırakılmaz, bir başka deyışle, eşzamanlı ıpucuyla ogretım yontemınde tum oğretım oturumlan sıfır sanıye bekleme surelı olarak gerçekleştunlır Daha once de değınıldığı gıbı, ę̧zamandı ıpucuyla ogretım yontemınde kontrol edıcı ıpucu her zaman ve ayırdedıcı uyarandan hemen sonra sunulduğu ıçın ogrrencıye bağımsız tepkı verme şansı verilmez, bu nedente de yoklama oturumları ogrretım oxurumlarından hemen once gerçekleştırılmelıdır (Gıbson ve Schuster, 1992)

Eşzamanlı upucuyla oğretım yontemının etkılı bır bıçımde uygulanabılmesı ıçın altı basamaklı bır model sunulmuştur (a) oğrencının tepkıde bulunması ıçın ıpıcu olacak uyaranı belırleme, (b) kontrol edicı ıpucunu belırleme, (c) tepkı aralığı suresını belırleme, (d) deneme ler arası sureyı belirleme, (e) ven kayıt yonteminı belırleme, (f) uygulama, kayıt etme ve oğrencının gosterdı̆̆ performansa gore uygulanan ogretım yontemmnde gereklt gonulen değtşıkıkJerı yapma

Eşzamanlı ıpucu oğretım yontemıne ılışkın yayınlanmış az sayıda çalışma vardır Eşzamanlı ıpucu oğretım yontemının okuloncesı çocuklara sozcuk okuma becerısınn oğgetılmesınde (Gıbson ve Schuster, 1992), gelışımsel gerlık gosteren okuloncesı donem çocuklanna yıyecek maddelerımun sozel olarak tanımlanmasının oğretılmesınde (MacFarland Smith ve diğ, 1993), orta derecede zlhunsel ozurlu ogrenctlere tabela yazılan okumasını oğretılmesınde (Sıngleton, Schuster ve Ault 1995) gıbı tek basarnakl akademık ve sosyal davranışların oğretıldığı gıbı dondurulmuş konsantreden meyve suyu hazurtama (Schuster ve Gnffen, 1993) gbo zıncırleme davranış ve becerler ogretılmektedır Ancak, bu yontemın değışık ozur ve yaş grubundakı kışılere tek 
basamaklı ve zıncırleme davranuşlann oğretı mındekı etkılıı̆ı̆ını ınceleyen çalışmalara gereksınım vardır

\section{g) Giderek Ipucunu Arttrrma Öğre- tim Yöntemi}

Yanlışsız oğretım yontemlennden bur digert olan ıpucunun giderek arttıldı ğ ogrtetum yontemınde dort yontemsel sureç vardır (Wolery

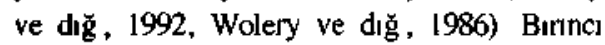
olarak, bu oğretım yontema en az uç duzeylı bır ıpucu hıyerarşısı gerekunır Ilk duzey oğrencının herhangı bır ıpucu almaksızın tepkude bulunması ıçın olanak tanınmasıdır, ıkıncı duzey ıse oğrencı davranşı uzennde en az kontrol ve en fazla kontrol gerekturen ipucu duzeyi arasinda yer alan aralıktur Hedef uyaran ilk upucu duze yınde sunulur ve oğrenciye kendısınden tepkide bulunması beklendığı anımsatılır (hıssettınlır) Ipucu hıyerarşısındekı uçuncu ve son aşama ıse ogrencının doğru tepkıde bulunmasını kesınleşturen kontrol edicı tpucunun sunulmasidir Ikıncı yontemsel sureç ıpucu hyerarşısı ıçınde her yardım duzeyınde becen yonergesı sunulmassdır Uçuncu yontemsel surę̧ oğretmen tarafından hıyerarşı ıçınde sunulacak olan ıpucunun sunulmasından once ve sonra oğrencının tepkıde bulunması ıçın bır tepkı aralıð̆ının brakılmasıdır Dorduncu ve son aşama ise ıpucu hıyerarşısı ıçınde hangı ıpucu duzeyınde gerçekleşırse gerçekleşsıı doğru oğrencı tepkılerının pekışırlmesıdır Ipucunun giderek artunlarak sunul duğg ogretım yontemınde ogretmen ya da uygulamact ogretume en az duzeyde ıpucu sunarak başlar Oğrencı doğru tepkude bulunursa davranışı pekıştınlır, doğnı tepkıde bułunmazsa hedef uyaran tekrarlanarak bur sonrakı upucu duzeyıyle yardım sunulur Bu ipucu duzeyınden sonra oğrencı doğnu tepkıde bulunursa oğretmen doğru tepkıyı pekıştınr, oğrencı doğru tepkıde bulunmazsa ołretmen hedef uyaranı sunduktan sonra bır sonrakı ıpucu duzeyım sunar Bu sureç oğrencıden beklenen davranıs dơ̛̆n bıçınde sergilenunceye değn devam ettıriır Bu oğretım yontemının temel amacı ogrenciden sergılemesı beklenen davramısıın kontrol edicı pucu sunulmadan once gerçekleşmesın sağlamaktır
Araşırma bulgulan ıpucunun giderek artınldığı oğretım yontemının hafif zuhınsel ozur (Martn, Cornuck, Hughes, Mullen ve Ducharme, 1984), orta ve tlen derece zihinsel ozur (Bates ve Renzaglıa, 1982, Coon, Vogelsberg ve Wullam, 1981. Demchak ve Browder, 1990, Gaule, Nietupskı ve Certo, 1985, Giangreco, 1983, Phillips, Rełd, Korabek ve Hursh, 1988), gorme ozuru (Correra, Poulson ve Salzberg, 1984) ve çok ozurtuluk (Le-Gnce ve Blampıed, 1997) gıbı değışık ozur grupla rında yer alan çocuklara oğretım yapmakta etkılı bır oğretım yontemı olduğuntı gostermışur

Gıderek ıpucunun artırıldığ oğretım yontemı bebeklerde (Correa ve dığ, 1984, Noonan, 1984) Ilkokul doneminde (Duker ve Michuelson, 1983), ortaokul-lise donemunde (Bates ve Renzaglia, 1982, Le-Grice ve Blampied, 1997, Phillıps ve diğ, 1988) ve yeuşkınlerde (Demhack ve Browder, 1990, Gaule ve dıð, 1985, Phullıps ve dığ, 1988) becen ve davranış oğretılmesınde de etkılı bır yontemdir

$\mathrm{Bu}$ oğretım yontemıyle çoğuntukla zıncırleme davranıs ve becerıler ogretılmışıs (Wolery ve dığ, 1992, Wolery ve diğ, 1986) Çamaşır ve kurutma makınası kullanımı (Cuvo, Jacobı ve Sıpko, 1981), bağımsız olarak dıs fıç̧alama becensı (Homer ve Kellıtz, 1975), gunluk yaşam becenlen (Demhack ve Browder, 1990), video kaset kaydetme ve bilgısayar kullanma (Le-Gnce ve Blamped, 1997), alı̧ venş yapma (Gaule ve diğ , 1985) gib zıncır leme becenlenn yanında alıcı dil becerısı (Phullıps ve di, , 1988), nesnelen alıcı ve ifade edici dil Ile tanimlama (Hupp, Mervis, Able ve Conroy-Gunter, 1986), oyun sirastnda ifade edıcı dıl kullanımı (Bates ve Renzaglıa, 1982) gıbı tek basamaklı becerilerın ogretımınde de etkılı olarak kullamimaktadır

Wolery ve digerlen (1992), giderek ıpucunun artırıldığ ơgretım yontemının etkılı bıçımde uygulanabılmesı ıçın aşağıda sıralanan sekız basamaklı oğretım plan onermışlerdır (a) ogrencımın tepkıde buluminası ıçın ıpucu olacak uyaran belırteme, (b) ıpucu hiyerarşısınde yer atacak olan upucu turlenu belırteme, (d) belir- 
lenen ıpucu turlenn ogrencı davranşı uzerınde en az kontrol gerektırenden en fazla kontrol gerektırene doğu sıralana, (e) tepkı aralığı suresın belırleme, (f) oğrencı tepkılenne sunulacak uygun tepkslen belurteme, (g) hedef davranışa bağı olarak uygun ven toplama sıstemın belırleme, (h) uygulama, kaydetme ve ogrencının gosterdığı performansa ılışkın değışıklıklerde bulunma

\section{h) Giderek İpucunu Azaltuma Ögretim}

\section{Yöntemi}

En yuksek yardımı sunma olarak da bilınen gıderek ıpucunu azalıma oğretım yontemı oğrencının doğru tepkıde bulunmassmı gerçekleştirecek en fazla yardım duzeyınde ıpucuyla oğretıme başlanarak zamanla oğretmentn ipucunu ortadan kaldırması olarak tanımlanır $\mathrm{Bu}$ oğretım yonternınde uç yontem sej sureç yer almaktadır Bırıncısı, oğretmen ya da uygulamacı hedef uyaran ve en az ıkı kontrol edıcı ıpucu belırier Belırlenen kontrol edıcı ıpucu ơ̆rencıden bekJenen olçut kaşsilanıncaya değın sunulur Olçuı karşılandıktan sonra ıse oğrencı davranışı uzerinde daha az kontrolu olan kontrol edıcı ıpucu sunulur Omeğin, yemek masası sılme becensinin ıpucunu giderek azaltma oğretım yontemıyle oğretıldı̆ı ogretım oturumlarında masayı sıimesı ıçın oğretmen ılkın oğrencınun elının ustunden tutarak oğrencının masayı sılmesını sağlar, bır sonrakı kontrol edıcı ipucu olarak oğrencının dırseğınden tutar ve daha sonra sadece tkı parmağıyla oğrencının bıleğınden tutarak masayı sılmesını sağlamak uzere kontrol edıcı ıpucunu sunabłlır Bu ogre tım yonteminde kontrol edicı ıpucu, Ipucu hıyerarşısı ıçınde datma ılk once sunulur Ikıncı olarak ıse, bır ıpucu duzeyınden dığerıne geçı s ıçın olçut belırientr Oğrencinın bır ıpucu duzeyınde ofçutu karşılamasılyła bırlıkte ogretmen, ogrencı davranışı uzensnde daha az kontrolu olan upucunu sunmaya başlar $\mathrm{Bu}$ sureç oğren cııın hedefi kaş̧ılamasına değın devam eder Uçuncu olarak ıse, oğretmen ởrencı davranışı uzennde daha az kontrole dayalı upucunun etksıtu değerlendırmek uzere yoklama vensı toplar Yoklama verısı toplamanın amacı oğrencının hedef davranışı daha az yardın duzeyıyle yerıne getırı getırmedığını araşırmakır (Wolery ve diğ, 1992, Wolery ve dið̆, 1986)

Araştırma bulgularn giderek ıpucunun azaltıldığı oğretım yontemının otızm (Duker ve Morsınk, 1984), hafif zuhınsel ozur (Kayser, B1llungsley ve Neel, 1986), orta ve Ilen derece zihurisel ozur (Colozzı ve Pollow, 1984, Curo, Leaf ve Borakove, 1978. Duker ve Michuelson, 1983, Rıchmond ve Lewallen, 1983) ve gelışımsel gerilık (Hınerman, Jenson, Walker ve Peterson, 1982) gıbı değışı ozur gruplarında yer alan çocuklara oğretım yapmakta etkılı bir oğretım yontemı olduğunu gostermıştır

Ipucunun gidereh azaluldığ oğretım yonteminin bebeklık doneminde (Dunts, Cushung ve Vance, 1985), Ilkokul doneminde (Luisell, Collozzı, Donellon, Helfen ve Pemberton, 1978), ortaokui-lıse doneminde (Wheeler, Ford, Nietupsk, Looms ve Brown, 1980) ve yeuşkınlerde (Cuvo ve dıg, 1981) becen ve davranus oğretumınde de etkılı olarak kullanıldığı gorulmektedır Ayrıca bu ogretım yontemıyle ışaret dılı kullanımı (Duker ve Mıchıelson, 1983), baş çevırme hareketı (Dunts ve dig, 1985), sorulan sorulan yantlama (Luisellı ve dı̆ , 1978), sozcuklerı ve hayvan ısımierinı sozel olarak tanimiama (Rıchmond ve Lewallen, 1983) gıbı tek basamakh becen ve davranışların oğretımınde olduğı kadar gyeceklenn turlerine gore katlanmass (Cuvo ve diğ, 1981), basıt ylyecek hazırlama (Kayser ve dığ, 1986) ve tuvalet temızleme (Cuvo ve dig, 1978) gıbı zıncırleme davranışların oğretımınde de etkulı bır ogretsm yontemı oldugu goruimektedir

Wolery ve diğerlen (1992), ipucunun gıderek azaltıldığ oğretım yontemının etkılı bıçımde uygulanabılmesı ıçın aşağıda sıralanan onbır basamaklı ogretım planun onermişjerdır (a) hedef davranış belırleme ve canımlama, (b) ogrencinun tepkıde bulunması ıçin ıpucu olacak uyaranı belırleme, (c) ıpucu hyerarşısınde yer alacak otan ıpucu duzey sayısin belırleme (d) ıpucu turlennı oğrencı davranışı uzerndekı kontrol etkısıne gore en çoktan en aza doğru sıralama, (e) ıpucu turlerını ogrrencı davranışı 
uzerinde fazla kontrol gerektırmestnden daha az kontrol gerektımmesıne doğru sıralama, (f) tepk aralı̌̆ı suresını belırleme, (g) oğrencı davranışı uzernde daha az kontrol gerekturen spucuna geçış olçutunu belırleme, (h) oğrencı davranışı uzennde daha az kontrol gerektıren spucunun sunulduğu ogrretım oturumlarında oğrencı performansını belırleyebılmek ıçın gerekıı değerlendıne planını belırleme, (ı) oğrencı tepklenne sunulacak uygun tepkilen belırleme, (j) hedef davranışa bağlı olarak uygun ven toplama sıstemını belırleme, (k) uygulama, kaydetme ve oğrencının gosterdı̆ performansa ılışkın değışıklıkerde bulunma

Yukarıda açıklanan oğrctınn yontemlen Ile ilgılı alanyazın ıncelendığınde bu ogretım yontemierının sadece ozel eğıtım oğretmenlen sınıf oğretmenlerı ve araştırmacıiar tarafından kullanıjmadığı, akranlara, anne-babalara ve kardeşlere yontemlenn nasıl uyguianabıleceğtnun oğretılerek bu kınselern yontem etkıil olarak kullanıp kullanmadıklarını araştıran çalışmalara da rastlanmıştır

\section{Sonuç ve Öneriler}

Ozet olarak yukarıda sunulan araştırmalardan yanlışsız oğretım yontemlerınden olan tepkı uçlaıının suntılduğu oğretım yontemlennın, değışık yaş ve gereksinum olan kışılere değışık becen ve davranışları kazandırmada etkılı yontemler oldukjan çıkarsamasını yapabılınz Bu oğretım yontemlennde hata oranının duşuk oiması, oğretmen ve oğrencı arasında olumlu etkıleşıme neden olması, hata oranı duşuk oldư̧̧u ıçın ox̆rencınun engellenme duygusunu yaşamasını en aza ındırdı̆̆ı ıçın ozel gereksınmm bureylere eğım sunmak uzere kullanumasi onenlebılır

Ayrica, ozel eğıtımde farklı uygułamalara değınılen gunumuzde, bu etkulı oğretım yaklaşımları uygulanarak (a) uygulamada karşılaşılan soruniara çozumler bulunabılır, (b) oğrenme ve ozretme surecı daha zevklı hale geturilebılır ve (c) yukanda venlen araşurıma bukgułarınunn daha guçlendırılmesıne Jolayısıyla, araştıma yontemlenne destek olunabılır

\section{KAYNAKÇA}

Alberto, PA, ve Troutman, AC (1995) Applied Behavior Analyss for Teachers (Fourth Ed.). New Jersey Memil Prentice Hall

Alı-Cybnivsky, C A, ve Schuster, J W (1990) Using constant ume delay procedures to teach muluplication facts Remedial and Special Education, 11 (1), $54-59$

Autt, M J, Wolery, M, Gast, D L, Doyle, PM, ve Eizenstat, V (1988) Comparison of response promptung procedures in teaching numericd identification to autstic subjects Journal of Autism and Developmental Disorders, 18, 627636

Ault, $M J$, Wolery, $M$, Gast, $D L$, Doyle, $P$ M, ve Martın, C P (1990) Compansion of predictable and unpredictable inal sequences during small-group insinution Learning Risability Quarterly, 13 (1), 12-29

Ваттег, R D, Lobato, Вапега, D, ve Sulzer Azaroff, B (1980) A sıtnujtaneous treatment comparson of three expressive language training programs with a mute autusuc chuld Journal of Autism and Bevelopmental Disorders, 10(1), 21 37

Ватега, R, ve Sulzer-Azaroff, B (1983) An alternatung treatment comparison of oral and total commumication tranıng programs with echolalsc dutıstic chuldren Journal of Appled Behavior Analysis, 16, 379-394

Bates, P, ve Renzaglia, A (1982) Language instruction with a profoundly retarded adotescent The use of a table game in the acquistion of verbal labelıng skills Education and the Treatment of Children, 5, 13-22

Browder, D M, Morns, W W, ve Snell, M E (1981) Using ume delay to teach manual signs to a severely retarded student Education and Training of Mentally Retarded, 16, 252-258

Charlop, M H, Schreibman, L, ve Thubodeaw, MG (1985) Increasing spontaneous verbal responding in autısuc children using a tıme delay procedure Journal of Applied Behavior Analysis, 18, 155-166

Cipan, E, Augustne, A ie Blomgren, E (1982) Teachung profoundly retarded adutts 
to ascend starrs safely Education and Training of the Mentally Retarded, 17 (1), 51-54

Cipan, E, ve Madigan K (1986) Errorless learnung Reserach and application for "difficult to teach" children Canadian Journal for Exceptional Children, 3(2), 39-43

Collıns B C, ve Stınson, D $M$ (1994-1995) Teaching generalızed reading of product waming labels to adolescents with mental disabilities through the use of key words Exceptionality, 5 (3), 163-181

Collozs, G A, ve Pollow, R S (1984) Teaching independent walking to mentally retarded chuldren in a public school Education and Training of the Mentally Retarded, 19 (2), $97-101$

Cooke, T P, ve Appollom, T (1976) Developing positive social-emotional behaviors A study of traning and generalization effects Journal of Applied Behavior Analysis, 9, 6578.

Coon, M E, Vogelsberg, R T, ve Willams, W (1981) Effects of classroom public transportation on generalization to the natural environment. Journal of the Association of the Severely Handicapped, 6 (2), 46-53

Cooper, O J, Heron, L. W, ve Heward, L. W (1987) Applied Rehavior Analysus. Ohuo Memill Publıshung Company

Correra, V I, Poutison ve Salzberg C L. (1984) Trainung and generalızation of research-grasp behavior in blind, retarded young chuldren Journal of Applied Behavior Analysis, 17, 57-69

Crouch, K P, Rusch, F R, ve Karlan, G R (1984) Competetuve employment Utilızing the correspondence trainıng paradigm to enhance productivity Education and Trainung of the Mentally Retarded, 19 (4), 268-275

Cuvo, A J, Jacobı, L, ve Sıpko, R (1981) Teaching laundry skills to mentally retarded students. Education and Trainung of the Mentally Retarded, 16 ( 1 ), 54-64

Cuvo, A J, Leaf, R B, ve Borakove, L. S (1978) Teachung jantonal skills to the mentally retarded Acqusition, generalization, and mantenance Journal of Applied Behavior Analysis, 11 (3), 345355

Deckner, C W, ve Blanton, R L. (1980) Classification of abnormal chuldren Discnmuation learming ability Journal of Autism and Developmental Disorders, 10 (4), 405-415.

Demhack, $M$, ve Browder, D $M$ (1990) An evaluation of the pyramıd model of staff training in group homes for adults with severe handicaps. Education and Training in Mental Retardation, 25 (2), 150-163

Doyle, P M, Schuster, J W, ve Meyers, S (1996) Embeddıng extra sumulı in the task direction Effects on leaming of students with moderate mental retardation Journal of Special Education, 29 (4), 38!-399

Duket, P C ve Morsınk, H (1984) Acquisition and cross-settıng generalization of manual signs with severly retarded chuldren Journal of Applied Behavior Analysis, 17 (1), 93-103

Duker, P C ve Michielson, H M (1983) Cross setung generalızation of manuel signs to verbal instructions with severely retarded chuldren Applied Research in Mental Retardation, 4 (1), 29-40

Dunts, C J, Cushing, P J, ve Vance, S D (1985) Response-contungent leaming in profoundly handicapped infants A social systems perspective Analysis and Intervention in Developmental Disabilities, 5 ( l/2), 33-47

Farmer, J A, Gast, D L., Wotery, M, ve Winterling, $V$ (1991), Small group instructoon for students with severe handicaps A study of observational leaming Education and Trainung in Mental Retardation, 26 (2), 190 201

Fink, W T, ve Bnce-Gray, K J (1979) The effcets of teaching strategles in the acquisition and recall of an academic task by moderately and severely retarded preschool chuldren Mental Retardation, 17, 8-12.

Frank, A R, ve Wacker, D P (1986) Analysts of a visual prompting procedure on acquistuon and generalization of con skills by 
mentally retarded childern American Journal of Mental Deficiency, 90(4), 468-472

Fredenck-Dugan, A, Test, D, ve Warn, L. (1991) Acquisition and generalization of purchasing skılls using a calculator by students who are mentally retarded Education and Training in Mental Retardation, 26 (4), 381-387

Gardill, M C ve Browder, D M (1995) Teaching stumulus classes to encourage independent purchasing by students with severe behavior disorders Education and Training in Mental Retardation and Developmental disabilities, 30(3), 254-264

Gast, D L, Doyle, P M, Wolery, M, Ault, M. J, ve Baklarz, J L. (1991) Acquisition of Incidental Informaton during small group instruction Education and Treatment of Children, 14( I), 1-8

Gaule, K, Nietupskı, J, ve Certo, N (1985) Teachung supernarkel shopping skills using an adaptove shopping list Education and Training of the Mentally Retarded, 20 (I), 53-59

Gee, K, Graham, N, Salor, W, ve Goelz, L. (1995) Use of integrated, general education, and communty setungs as promary contexts for skill instruction for studenets with severe, multuple disabilities Behavior Modification, 19(I), 33-58

Giangreco, M F (1983) Teaching basic photography skulls to d severely handcapped young adult using simulated matenals Journal of the Association of the Severely Handicapped, 8 (3), 43-49

Gibson, A N, ve Schuster, J W (1992) The use of simultaneous promptıng for teaching expressive word recognitıon to preschool chldren Topics in Early Childhood and Special Education, 12(2), 247267

Goetz, L, Gee, K, ve Salor, W (1983) Crossmodal transfer of stımulus control Preparing students with severe multuple disabylitues for audiological assessment Journal of the Association for Persons with Severe Handicaps, 8(4), 3-13

Hardırıan, S A, Goetz, E. M, Reuter, K E, ve LeBlanc, J M (1975) Pnmes, contungent attention, and tranıng Effects on a chuld's motor behavior Journal of Applied Behavior Analysis, 8, 399-409

Hinerman, P S , Jenson, W R, Walker, G R, ve Peterson, P B (1982) Posttive prac ice overcorrection combined with additional procedures to teach signed words to an autisitc child Journal of Autism and Developmental Disorders, 12(3), 253-263

Hormer, R W, ve Keilitz, I (1975) Training mentally retarded adolescents to brush their teeth Journal of Applied Behavior Analysis, 8, 301-309

Hupp, S C, Mervis, C B, Able, H, ve Conroy-Gunter, M (1986) Effects of receptive and expressive training of category labels on generalızed learnung by severely mentally retarded chuldren American Journal of Mental Deficiency, 90, 558-565

Katz, S, Goldberg, J, ve Shurka, E. (1977) The use of operant techniques in teaching severely retarded clients work habits. Education and Training of the Mentally Retarded, 12, 1420

Kayser, J E., Billıngsley, F F, ve Neel, R. S (1986) A companson of m-context and traditional instnuctional approaches Total task, single trial versus backward channing, multiple trals. Journal of the Association for Persons with Severe Handicaps, 11 (1), 28-38

Kleınert, H L., ve Gast, D L. (1982) Teaching a multıply handicapped adult manual signs using a constant ume delay procedure Journal of the Association for the Severely Handicapped, 6, 2532

Knapczyk, D R (1983) Use of teacher-paced instruction in developing and mantaIning independent self-feeding Journal of the Association for the Severely Handicapped, 8, (3), 10-16

Koegel, R. L., Dunlap, G , Rıchman, G $S$, ve Dyer, K (198I) The use of specific orientung cue for teachung discrimunation tasks Analysis and Intervention in Developmental Disabilities, 1, 187198.

Koegel, R L, ve Rincover, A (1977) Research on the difference between generalizatoon and maintenance in extra-therapy responding Journal of Applied Behavior Analysis, 10(1), 1-12 
Le-Gnce, $B$, ve Blampied, $N M$ (1997) Learning to use video recorders and personal computers with increasing assistance prompting Journal of Developmental and Physical Disabulities, $9(1), 1730$

Luciano, M C (1986) Acquisition, maintenance, and generalızation of productive intravertal behavior through transfer of stumulus control procedures Applied Research in Mental Retardation, 7(1), 1-20

Lusseli, J K, Colloz, G, Donellon, S. Helfen, C S, ve Pemberton, B W (1978) Traıning and generalızation of a greeting exchange with a mentally retarded, langungedeficient chuld Education and Treatment of Children, 1(4), 23-29

MacDuff, G S, Krantz, P J, ve McClannahan, L. E (1993) Teaching chidiren with autism to use photographc actuvity schedules Mantenance and generalızation of complex response cham Journal of Applied Behavior Analysss, 26(1), 8997

MacFartand Smtth, J, Schuster, J W. ve Stevens, K (1993) Using simultaneous prompting to teach expressive object identuficauon to preschoolers with developmental disabılities Journal of Early Intervention, 17(1), $50-60$

Marchiett, A G, McCartney, J R, Drain, S, Hooper, M, ve Dix, J (1983) Pedestnan skilis training for mentally retarded adults Companson of trainng in two settings Mental Retardation, 21(3), 107-110

Martın, G, Comıck, G, Hughes, J, Mullen, H, ve Ducharme, D (1984) Trauning derelopmentally handicapped persons as supervisors in sheltered workshops Applied Research in Mental Retardation, 5, 199-218.

Mattıngly, J C se Bott, D A (1990) Teaching muluplication facts to students with leamıng problems Exceptional Chuldren, 56 (5) $438-449$

McDonnell, J, ve Ferguson, B (1989) A companson of tume delay and decreasing prompl herarchy strategies in teachtng banking skills to students with moderate handicaps Journal of Applied Behavioral Analysis, 22, 85-91
McGee, $G$ G, Krantz, $P$ J, ve McClannahan, L. E. (1984) Conversational skılls for autustuc adolescents Teaching assertı veness in naturalıstic game settıngs Journal of Autism and Developmental Disorders, 14, $319-330$

Mcllvane, W J, Wthstandley, J K, ve Stoddard, L. T (1984) Postuve and negative stumulus relatıons in severely retarded individu als' conditıonal discrimınation Analysis and Intervention in Developmental Disabilittes, 4, 235251

McKelvey, J L., Sisson, L. A, VanHasselt, V B ve Hersen, M (1992) An approach to teaching self-dressing to a cheld with dual sensory imparment. Teaching Exceptional Chlidren, 25(1), 12-15

Noonan, M J (1984) Teaching postural reactions to students with severe cerebral palsy An evaluation and theory and technique The Journal of the Association for Persons with Severe Handicaps, 9, I11-122

Paisey, T J, Whitney, R B, ve Moore, J (1989) Person treatment intercations across nonaversive response-deceleration procedures for self-ınjury A case study of effects and side effects Behavioral-Residential-Treatment, 4 (2), 69-88

Peterson, G A, Austın, G J, ve Lang, R P (1979) Use of teacher prompts to increase social behavıor Generalızation effects with severely and profoundly retarded adolescents. American Journal of Mental Deficiency, 84, 82-86

Phillips, J F, Reid, D H, Korabek, C. A, ve Hursh, D E. (1988) Communily-based instruction with profundly mentaliy retarded persons Client and public responsiveness. Research in Developmental Disabilities, $9(1)$, 3-21

Reese G M, ve Snell, M E. (1991). Puttung on and removing coats and jackets The acquistion and maintenance of skılls by children with severe multuple disabilutues Education and Traning in Mental Retardation, 26(4), 398. 410

Richmond, G, ve Lewallen, J (1983) Facilitating transfer of sumulus control when :eaching verbal labels Education and Trainung of the Mentally Retarded, 18(2), 111-116. 
Rowan, V C., ve Pear, J J (1985) A companson of the effects of interpersonal and concurrent training sequels on acquisition, retention and generalızation of pictures names Applied Research in Mental Retardation, 6, 127-145

Sanato, D M, Strain, P S, Lefebvre, D, ve Rapp, N (1987) Facilitatung transtion tumes with handicapped chidren A comparison between peer-mediated and antecedent prompt procedures Journal of Applied Behavior Analysis, 20 (3), 285-291

Salısbury, C. Wambold, C, ve Walter, G (1978) Manual communication for the severely handicapped An assessment and instructional strategy Education and Training of the Mentally Retarded, 13(4), 393-396.

Schloss, P, Alexander, N, Harning, E. ve Wrigth, B (1993) Teaching meal preparatjon vocabulary and procedures to individuals with mental retardation Teaching Exceptional Children, 25(3), 7-12

Schoen, S F (1986) Assistance proce dures to facilisate the transfer of stimulus control Review and analysis Education and Training of the Mentally Retarded, 21(1), 274

Schoen, S F, ve Sivl, E O (1989) A comparison of procedures in teaching selfhelp skulls Increasing asststance, tome delay and observational learrung. Journal of Autism and Der elopmental Disorders, 19, 57-72

Schuster, J W, Gast, D L., Wolery, $M$, ve Guilunan, $S$ (1988) The effectiveness of constant ume-delay procedure to teach chal ned responses to adolescents with mental retardation Journal of Applied Behavioral Analysis, 21, 169-178.

Schuster, J W, Griffen, A K, ve Wolery, M (1992) Comparison of simultaneous prompting and constant ume delay procedu res in teaching sight words to elementary students with moderate mental retardation Journal of Behavioral Education, 2(3), 305325

Schuster, J W, Stevens, K B, ve Doak, P K (1990) Using constant tıme delay to teach word definutions The Journal of Special Education, 20(3), 306-318.

Schuster, J W, ve Griffen A K
(1990) Using time delay with task analysis. Teaching Exceptional Children, Summer, 49.53

Schuster, J W, ve Groffen, A K (1991) Using constant time delay to teach recıpe following skılls Education and Training in Mental Retardation, December, $411-419$

Schuster, J W, ve Griffen, A K. (1993) Usıng a sımultaneous promptıng strategy to teach a chained task to elementary students with moderate mental retardation Journal of Behavioral Education, 3(3), 299-315

Shapro, E. S, ve Shendan, C A (1985) Systematic assessment and trainng of sex education for a mentally retarded wornan Applied Research in Mental Retardation, 6, $307-317$

Sindelar, $P$ T, Bursuck, W $D$, ve Halle, J W (1986) The effects of two varations of teacher questionng on student performance Education and Treatment of Children, 9, 56-66

Sungh, $N \mathrm{~N}$, ve Milichamp, C J (1987) Independent and social play among profoundly mentally retarded adults Traunng, mantenance, generalızation, and long-term follow-up Journal of Applied Behavior Analysis, 20( 1 ), 23-34

Singleton, K C, Schuster, J $W$, ve Ault, M. J (1995) Simultaneous prompting in a small group instructional ajrangement Education and Training in Mental Retardation and Developmental Disabilities, September, 218-230

Sisson, L A, Kliweın, M L, VanHasselt, V B (1988) A graduated gundance procedure for teaching self dressing skulls to multhandicapped chuldren Research in Developmental Disabjilities, 9(4), 419-432

Smith, M, ve Meyers, A (1979) Telephone-skulls training for retarded adults Group and individual demonstrations with and without verbal instruction American Journal of Mental Deficiency, 83, 581-587

Snell, M S (1982). Analysıs of tıme delay procedures in teaching datly lıving skılls to retarded adults Analysis and Interventron in 
Developmental Disabilities, 2, 139-155

Stenberg, L, McNerney, C D, ve Pegnatore, L, (1985) Developing co-active Imitative behaviors with profoundly mentally handicapped students Education and Training of the Mentally Retarded, 20(4), 260-267

Slevens, K, ve Schuster, J W (1987) Effecis of a constant tume delay procedure on the writlen perfornance of a learning disabied student. Learning Disability Quarterly, 10, 9. 16

Stımbert, V E, Mınor, J W, ve McCoy, J F (1977) Intensıve feedıng traunıng with retarded children Behavior Modification, 1(4), 517-530

Venn, M L., Wolery, M, Werts, M G , ve Morns, A (1993) Embedding insiruction in art activittes to teach preschoolers with disabiluties to imitate their peers. Early Childhood Research Quarterly, 8(3), 277-294

Wacker, D P, ve Greenbaum, F T (1984) Efficacy of a verbal training sequence on the sortung performance of moderately and severely retarded adolescents American Journal of Mental Deficiency, 88, 653-660

Walls, R T , Haught, P, ve Dowler, D L (1982) Moments of transfer of stimulus control in practical assembiy tasks by mentally retarded adults. Americap Journal of Mental Deficiency, 87, 309-315

Welch, J, Nietupski, J, ve HamtreNietupsk, S (1985) Teaching publıc transportatson problem solving skills to young adults with moderate handicaps Education and Training of the Mentally Retarded, 20, 287295

Wheeler, J, Ford, A, Nietupskı, J, Looms, R, ve Brown, L. (1980) Teaching moderately and severely handicapped adolescents to shop in supermarkets using pocket calculators Education and Training of the Mentally Retarded, 15(2), 105-l I2.

Whitman, $T$ L., Zakaras, $M$, ve Chardos. S (1971) Effects of remforcement and guidance procedures on instruction-following behavior of severely retarded children Journal of Applied Behavior Analysis, 4(4), 283290

Wolery, M, Auit, M J, Doyle, P M, ve Gast, D L (1986) Comparison of Instructional Strategies: A Iterature Review. (U S Department of Education, Grant \# G008530197) Lexington, KX Department of Special Education

Wolery, M, Ault, M J, ve Doyle, P M (1992) Teaching Students with Moderate to Severe Disabrlities: Use of Response Prompting Strategies. NY Longman Pubłshung Group

Wolery, M, Balley, D B , ve Sugar, G $M$ (1988) Effectuve Teaching: Principles and Procedures of Applied Behavioral Analysis with Exceptuonal Students. Boston Allyn ve Bacon, linc

Wolery, M, Gast, D L., Kırk, K, ve Schuster, J. (1988) Fadıng extra-stımulus prompts with autustic chuldren using time delay Education and Treatment of Chldren, 11, 29-44

Young, K R, West, R P, Howard, V $F$, ve Whitney, $R$ (1986) Acquisition, fluency traıning, generalızation, and mantenance of dressing skills of two developmentally disabled ctuidren Education and Treatment of Children, 9(1), 16-29

Zane, $T$, Handen, B L., Mason, S A, ve Geffin, C (1984) Teaching symbol idenufication A comparison between standard promptıng and intervening response procedures Analysis and Intervention in Developmental Disabilities, 4(4), 367-377 